Case Report

\title{
Non operative management of superior mesenteric and portal vein thrombosis
}

\begin{abstract}
Acute mesenteric ischemia refers to the sudden onset of intestinal hypoperfusion, one cause of which can be mesenteric venous occlusion. Acute superior mesenteric vein (SMV) and portal vein (PV) thrombosis can be a complication of hypercoagulable, inflammatory, or infectious states. It can also occur as a complication of medical or surgical intervention. Management of mesenteric and portal vein thrombosis includes both operative and nonoperative approaches. Operative interventions include thrombectomy with thrombolysis; this is often employed for patients who present with signs of peritoneal irritation. Nonoperative approaches can be either noninvasive or invasive. Treatment with anticoagulation has been shown to be efficacious, and recanalization can be achieved (Figure 1)
\end{abstract}

Volume 8 Issue 2 - 2017

\section{Ziad Alhariri}

Emergency Medicine, Qatar

Correspondence: Ziad Alhariri, Emergency Medicine, HMC, SBEM, Doha, Qatar, Email zdalhariri@yahoo.com

Received: May 14, 2017 | Published: June 09, 2017

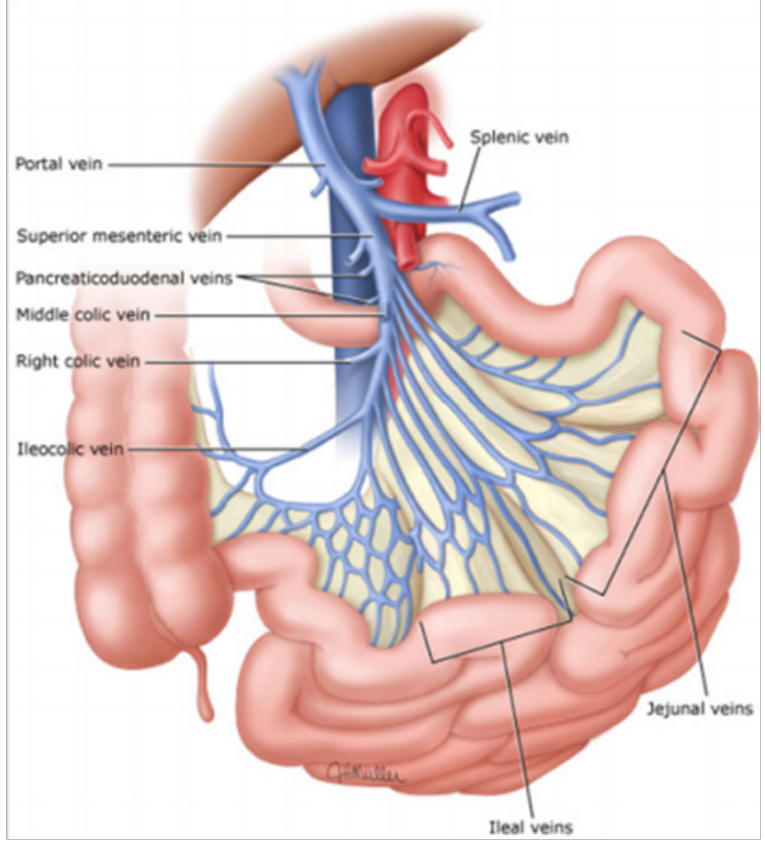

Figure I $N=57$; Epidemiological distribution of the pathological fractures, traumatic fractures, and nonunion.

\section{Case presentation}

50-year-old gentleman previously healthy, non smoker, non alcoholic presents to Emergency Department (ED) with left hypochondrial colicky non radiating pain, aggravated by heavy meal for five days, and associated with severe diaphoresis and nausea. He sought for medical advice twice and treated first time as a case of gastritis, and on second visit basic blood work including $\mathrm{CBC}, \mathrm{U \& E}$, Lactate, LFTs were within normal range and Ultrasound showed no abnormalities, and discharged on Paracetamol and antacids. Patient did not improve inspite of compliance with the prescribed medications and presented to our ED by the same complaints, on examination, patient was stable vitally, with soft non-tender abdomen and normal bowel sounds. He underwent comprehensive study including $\mathrm{Ct}$ abdomen with contrast which reveled: Thrombosis of the superior mesenteric vein and its branches noted. The thrombus is seen extending, through the main portal vein partially occluding its lumen, to involve the splenic vein and right portal vein as well (Figure 2). Enoxaparin was started for him, work up for thrombophilia was sent, GI consultation was made to arrange for OGD (Oesophago-gastro-Dudenoscopy), looking for malignancy which revealed erosive gastritis with significant modularity, CLO test was negative, tumor markers were sent (CA 125- CA19-9 CEA) were normal, homocysteine 13.9 (high), $\mathrm{B} 12=111$ (low), during his stay at hospital patient has no complain, and his physical exam was normal.

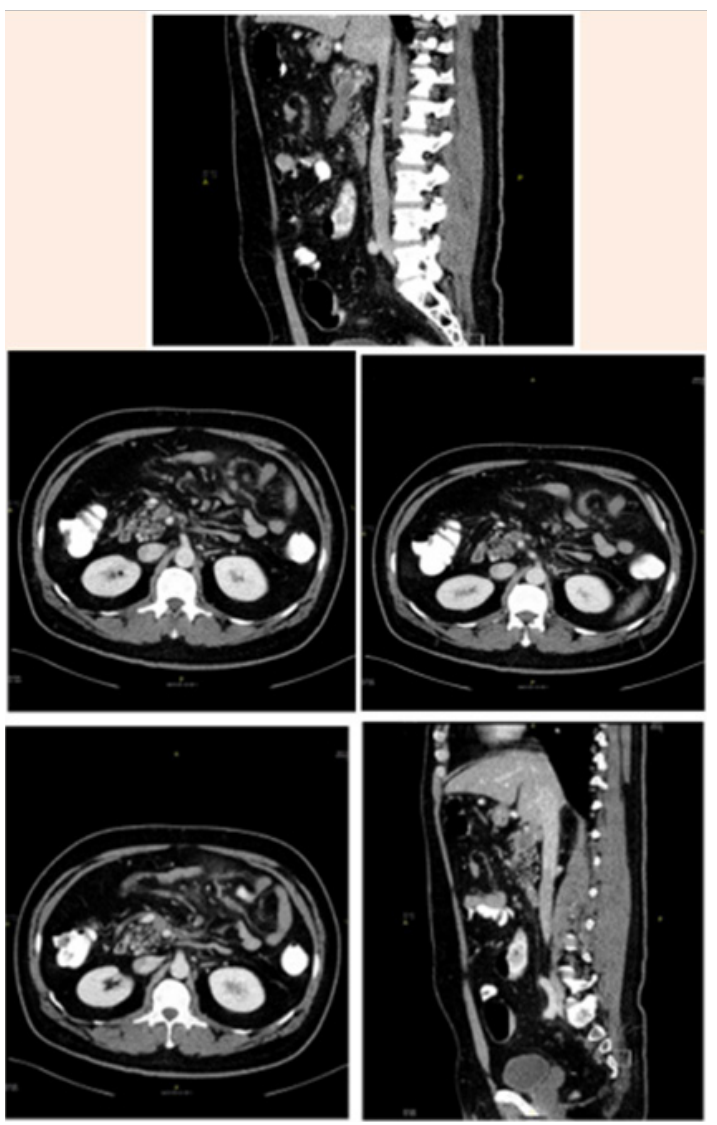

Figure $2 \mathrm{~N}=57$; Epidemiological distribution of the pathological fractures, traumatic fractures, and nonunion. 


\section{Treatment}

Enoxaparin $60 \mathrm{mg}+40 \mathrm{mg}$ subcutaneous, $\mathrm{q} 12 \mathrm{~h}$

Folic acid $5 \mathrm{mg}$, orally - daily

Pantaprazole $40 \mathrm{mg}$, rally - daily

Vitamin B12 (cyanocobalamin) 1mg / $1 \mathrm{ml}$, inj, IM, daily vitamin B6 (pyridoxine) $40 \mathrm{mg}$, tab, oral, daily.

\section{Outcome and follow up}

Patient discharged from hospital with diagnosis of homocystienemia, B12 deficiency, and normal thrombophilia work up. With plan to start rivaroxiban 20mg PO OD, frequent follow-up at clinic. After 6 month ct abdomen with contrast repeated and revealed recanalization of the portal vein and splenic vein with partially recanalization of the superior mesenteric vein which is surrounded with fat standing and multiple sub centimeter of lymph nodes.

\section{Discussion}

Nonoperative management of acute MVT is feasible when the diagnosis made by means of the initial CT scan is certain and when bowel infarction has not led to transmural necrosis and bowel perforation. Hospital stay is shorter when patient treated non operatively, thrombosis is reversible with anticoagulation alone. ${ }^{1-4}$

\section{Conclusion (Learning points)}

The diagnosis of acute MVT was established either if, There was a positive CT scan result or when the patient Underwent laparotomy.
A CT scan was considered to be positive for MVT when a thrombus in the superior mesenteric. Recently, nonoperative treatment has been proposed in patients with MVT, We changed our preferred treatment from surgical to nonoperative management.

\section{Acknowledgements}

None.

\section{Conflicts of interest}

The authors declare that there is no conflict of interest.

\section{References}

1. Condat B, Pessione F, Helene Denninger M, et al. Recent portal or mesenteric venous thrombosis: increased recognition and frequent recanalization on anticoagulant therapy. Hepatology. 200;32(3):466470 .

2. Primignani M. Portal vein thrombosis, revisited. Dig Liver Dis. 2010;42(3):163-170.

3. Subramanyam BR, Balthazar EJ, Lefleur RS, et al. Portal venous thrombosis: correlative analysis of sonography, CT and angiography. Am J Gastroenterol. 1984;9(10):773-776.

4. Catalano OA, Choy G, Zhu A, et al. Differentiation of malignant thrombus from bland thrombus of the portal vein in patients with hepatocellular carcinoma: application of diffusion-weighted MR imaging. Radiology. 2010;54(1):154-162. 